\title{
Persistent directional cell migration requires ion transport proteins as direction sensors and membrane potential differences in order to maintain directedness
}

\author{
Nurdan Özkucur ${ }^{*}$, Srikanth Perike ${ }^{\dagger}$, Priyanka Sharma, Richard HW Funk
}

\begin{abstract}
Background: Ion transport proteins generate small electric fields that can induce directional cell motility; however, little is known about their mechanisms that lead to directedness. We investigated $\mathrm{Na}$, K-ATPase (NaKA) and $\mathrm{Na}+/ \mathrm{H}+$ exchanger isoforms (NHE1 and 3) in SaOS-2 and Calvarial osteoblasts, which present anode- and cathode- directed motility, during electrotaxis.

Results: Significant colocalizations of NaKA with vinculin and pNHE3 with B-actin were observed to occur at the leading edges of cells. The directedness were attenuated when NaKA or NHE3 was inhibited, confirming their implication in directional sensing. Depending on the perceived direction, a divergent regulation in PIP2 levels as a function of NHE3 and NaKA levels was observed, suggesting that PIP2 may act as a spatiotemporal regulator of the cell membrane during electrotaxis. Moreover, at the same places where pNHE3 accumulates, bubble-shaped $\mathrm{H}^{+}$ clouds were observed, suggesting a physio-mechanical role for NHE3. The cell membrane becomes hyperpolarized at the front and depolarized at the back, which confirms NaKA activity at the leading edge.
\end{abstract}

Conclusion: We suggest a novel role for both NaKA and NHE3 that extends beyond ion translocation and conclude that they can act as directional sensors and $V_{\text {mem }}$ as a regulatory cue which maintain the persistent direction in electrotaxis.

\section{Background}

Directional cell motility plays an essential role in many biological processes, such as tissue formation/regeneration, wound healing, or tumor metastasis, and can be induced by both endogenously occurring and externally applied electric fields (EF). Persistent directionality requires precise, dynamic and regularly repeated cycles of interactions between cytoskeleton proteins, cell membranes, and the extracellular matrix so as to promote de novo protrusions at the leading edge of migrating cells, which distinguishes this process from random cell movement [1]. Focal adhesion complexes at the cell membrane-matrix interface allow for forward cell locomotion through rapid protein turnover, which results in

\footnotetext{
* Correspondence: Nurdan.Oezkucur@tu-dresden.de

+ Contributed equally

Department of Anatomy, Medical Faculty Carl Gustav Carus, Technical University of Dresden, Dresden, Germany
}

a dynamic connection/disconnection to the matrix as cells move in a preferred direction. Thus, proteins that are preferentially located at these sites during directional movement may play a significant role in direction sensing. Previous works, which have investigated different cell types, have observed that many proteins, lipids, and organelles redistribute because cells are polarized during electrotaxis [2-4]; however, the molecules and mechanisms that enable cells to perceive direction during electrotactic, persistent motility have yet to be investigated.

Apart from the many studies that have investigated the cytoskeleton proteins that modulate cellular migration machinery, recent studies have focused on membrane ion transporters and their interactions with cytoskeleton proteins [5-7], specifically in their potential roles in cell motility. The emerging roles of membrane ion transport proteins in the control of electrotaxis and in directionally persistent cell migration have first been 
reported for the voltage-gated $\mathrm{Na}^{+}$channel in rat prostate cancer cells [8], followed by other studies concerning PKD2 cation channels in directional sperm movement [9], aquaporins in astroglial cell migration [10], potassium channels in the invasiveness of embryonic stem cells [11], TRP channels in embryonic lung fibroblast motility [12], and a recent report from our own work concerning voltage-gated calcium channels in the electrotaxis of osteoblast cells [13].

Aside from ion translocation, several recent studies have reported on the roles of both NHE and NaKA in cytoskeletal remodeling, cell polarity, and lamellipodia formation [14-18]. When taken together, especially in regard to their primary functions as $\mathrm{pH}$ or cell volume regulators and cellular migration-specific membrane potential state modulators, NHE and NaKA can provide new insights into the understanding of both the physiological and the mechanical regulation of directional sensing in cells.

Herein, we suggest that NaKA and NHE3 can act as directional sensors in EF-induced directional cell motility via a mechanism that involves PIP2 as a potential mediator and the cell membrane potential $\left(\mathrm{V}_{\text {mem }}\right)$ as a regulatory cue. Using SaOS-2 and Calvarial osteoblasts, which represent anode- and cathode- directed motility, respectively, we show that 1 ) active NHE3 is concentrated in membrane protrusions that are accompanied by proton fluxes $\left(\mathrm{pH}_{\mathrm{i}}\right)$ at the leading edge of the cellular migration, especially in cathode-directed cells, and its activity is required for the perception of direction; 2) NHE1 is homogenously localized throughout the surface membrane and is involved in directional migration; 3 ) $\mathrm{V}_{\text {mem }}$, as a result of NaKA activity, has a regulatory function that maintains the persistent directionality by modulating the spatiotemporal changes between the leading edge (hyperpolarized) and the rear end (depolarized) in directionally migrating cells.

\section{Results}

NHE3 and NaKA colocalize with ß-actin and vinculin on the leading-edge of the cell

Phosphorylated NHE3 (pNHE3) was observed to specifically accumulate at the membrane protrusions of polarized $(\mathrm{EF})$ and non-polarized (control) cells. Interestingly, pNHE3 only appeared at the same places where $\beta$-actin accumulates, which represents significant colocalization at cell leading-protrusions in both anode (SaOS-2)- and cathode (Calvaria)-directed cells (Figure 1A). The colocalization rate of $\mathrm{pNHE} / \mathrm{B}$-actin was observed to increase by $30.4 \%$ and by $9.8 \%$ in cathode- and anode- directed cells, respectively, during electrotaxis (Figure $2 \mathrm{~A}$ ). The total NHE3 was evenly distributed on the cell membrane with less significant accumulations in the protrusions (Additional file 1). NHE1 exhibited a homogenous distribution throughout the cell membrane, regardless of cell polarization or migration direction (Additional file 2).

Unlike NHE3, the total NaKA was observed to specifically accumulate at the membrane protrusions of polarized and non-polarized cells. The pattern of accumulated $\mathrm{NaKA}$, which was especially striking in calvaria cells, resembled that of the focal adhesion marker protein, vinculin, which directed us to further investigate whether $\mathrm{NaKA}$ and vinculin are colocalized in cell membrane protrusions. Indeed, significant colocalizations of NaKA with vinculin were observed at the cell periphery of nonpolarized cells and at the leading edge of polarized cells during both cathodal (Calvaria) and anodal (SaOS-2) cell motility. Additionally, NaKA was localized also at the cell nuclei (Figure 1B). Very slight decreases of $0.1 \%$ and $1.4 \%$ in cathode- and anode-directed cells, respectively, in the colocalization rate of $\mathrm{NaKA} /$ vinculin were observed during electrotaxis (Figure 2A). Phosphorylated NaKA was homogenously distributed on the cell membrane during directed motility (Additional file 3).

\section{$\mathrm{EF}$ induces the relocation of proteins to the cell leading- protrusions}

In order to define the degree of protein relocation to the leading edge during directed cell movement, we plotted the distribution of each protein as a ratio of the mean fluorescence intensity in protrusions to that of the cytoplasm in polarized and in non-polarized cells. The fluorescence intensity ratios for the total NaKA, vinculin, and $B$-actin were greater in polarized cells than in nonpolarized cells, which reveal that these proteins were relocated from the cytoplasm to the membrane protrusions during directional cell movement. In both cathode- and anode-directed cells, relocation rates of NaKA from cytoplasm to cell leading edge were enhanced by $83 \%$ and $127 \%$, respectively, during electrotaxis. In cathode-directed cells, relocation rate of vinculin and $B$-actin to the leading edge of the cell was enhanced by 300 and $400 \%$, respectively, whereas in anode-directed cells, vinculin and $B$-actin were relocated by only $82 \%$ and $19 \%$, respectively; however, the relocation of pNHE3 to the leading edge was enhanced by $400 \%$ in cathode (Calvaria)-directed cells, whereas it was diminished by $41 \%$ in anode ( $\mathrm{SaOS}-2)$-directed cells (Figure 2B). PIP2 was observed to be located along the cell periphery during both cathodal and anodal movement; however, it was also located along the cell periphery in randomly migrating cells (Additional file 4).

\section{Cellular directedness is regulated by the activity of NHE3, NHE1 and NaKA}

EF-induced cellular directedness was attenuated when NHE3, NHE1, or NaKA activity was suppressed via the use of the specific inhibitors, S3226, HOE 642 or Oubain, 


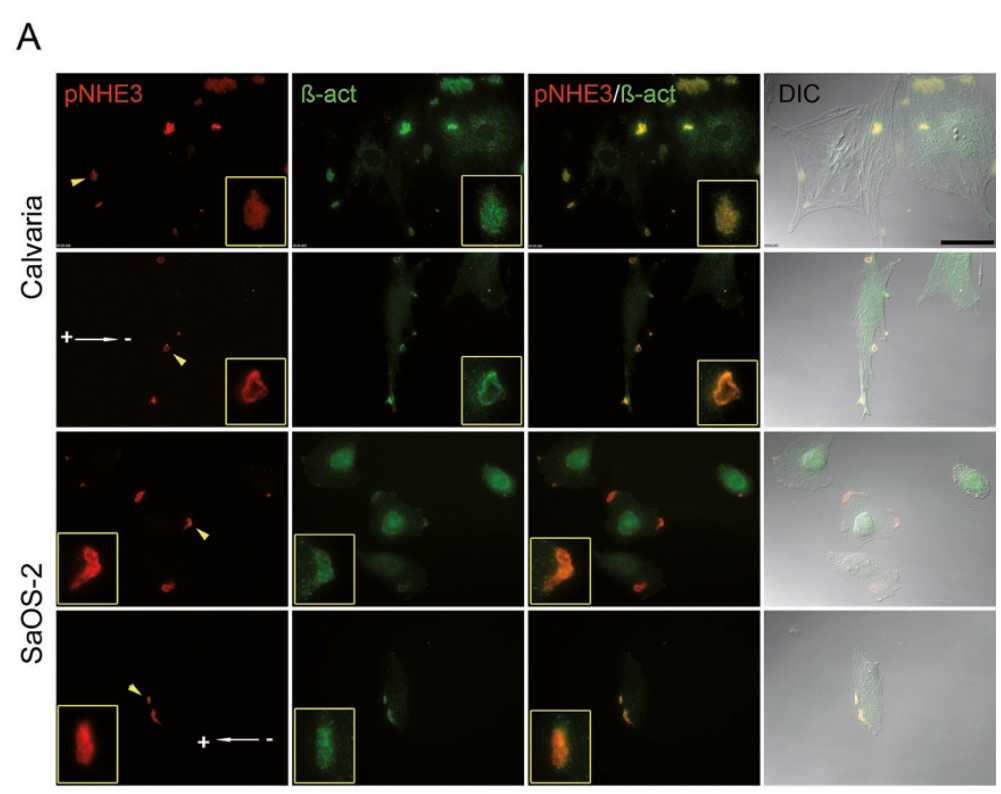

B



Figure 1 The localization of pNHE3 and of NaKA in EF-directed- versus random cellular movement. The colocalization of pNHE3 with $B$ actin (A) and of NaKA with vinculin (B) on the leading edge of a cell in both cathode (Calvaria)- and anode (SaOS-2)-directed cells. First and third row: randomly migrating control cells. Second and fourth row: polarized cells that persist in a direction under an applied EF. White arrows with " + " and "-" poles indicate the migration direction under an applied EF. Yellow arrows indicate the magnified view and correspond to the entire row. Bar: $50 \mu \mathrm{m}$.

respectively, in both cathode- and anhode-directed movement. Cells that were treated with the FAK inhibitor, PF573228, were used as a positive control. Although the cells exhibited an elongated shape in the applied EF as normal, they failed to form a polarized shape, which is characterized by a well-defined leading edge and rear end, when NHE3, NHE1, or NaKA was inhibited (Figure 3).
The inhibition of NHE3, NHE1, or NaKA slightly affected (a maximum of 50\%) the net displacement of both cathode- or anode- directed cells. In contrast, especially in anode-directed cells, inhibition dramatically affected the cellular speed. SaOS-2 cells exhibited a $300 \%$ increased speed when NHE3 was inhibited $(3 \mu \mathrm{M}$ S3226) and a 500\% increased speed when NHE1 was 



Figure 2 Graphics showing the colocalization rate and relocation of proteins during electrotaxis. The colocalization rates of pNHE3/Bactin and of NaKA/vinculin with and without EF exposure in cathode (Calvaria)- and anode (SaOS-2)-directed cells (A). The EF-induced relocation of pNHE3, NaKA, B-actin, and of vinculin to or from the leading edge of a cell during cathode (Calvaria)- and anode (SaOS-2)-directed motility (B). Error bars represent the SEM for $16-31$ cells from three separate experiments $(* P<0.05$ compared to the control -wothout EF-).

inhibited (using $10 \mu \mathrm{M}$ HOE 642). In cathode-directed cells, the pharmacological inhibition of NHE3 and NHE1 resulted in an average increase of only $21.5 \%$ and $25.3 \%$, respectively. The inhibition of NaKA divergently affected the cellular speed in cathode- and in anodedirected cells. The cellular speed was increased by $67 \%$ (using $20 \mathrm{nM}$ Oubain) in cathode- and decreased by $71 \%$ (using $1 \mathrm{nM}$ Oubain) in anode-directed cells when NaKA was inhibited. The inhibition of FAK, which served as a positive control in this study, caused a $500 \%$ (using $0.2 \mu \mathrm{M}$ PF573328) increase in cellular speed in anode-directed cells, whereas the speed of cathodedirected cells increased only by $48 \%$ (using $1 \mu \mathrm{M}$ PF573328) (Figure 4).

The intracellular levels of pNHE3 which are associated with PIP2, are divergently regulated depending on the perceived direction during electrotaxis

The intracellular levels of both total and phosphorylated NHE3 decreased by 7\%, which was also associated with a 21\% decrease in PIP2 levels, in cathodally migrating cells (Calvaria). In contrast, the total and phosphorylated
NaKA levels were elevated by $17 \%$ and by $12 \%$, respectively, in cathode-directed cells. The NHE1 level was not affected during cathodal electrotaxis (Figures 5A and $5 \mathrm{C}$ ). In anodally migrating cells (SaOS-2), the intracellular level of phosphorylated NHE3 increased by $29 \%$ whereas the total NHE3 level was not affected. Furthermore, the PIP2 level in anode-directed cells was also increased by $7 \%$. Similar to what was observed for NaKA levels in cathode-directed cells, phosphorylated NaKA levels also increased by $23 \%$ in anode-directed cells, but without a change to the total NaKA levels. The NHE1 level decreased by $7 \%$ during anodal electrotaxis (Figures 5B and 5D).

The cell membrane is relatively hyperpolarized at the leading edge versus the rear end during directed migration

Regardless of migration direction, different membrane potential at two ends of the cell was observed, that is, in both cathode- and anode-directed cells; the cell membrane exhibited a relatively hyperpolarized state on the leading edge in comparison to the rear end during 


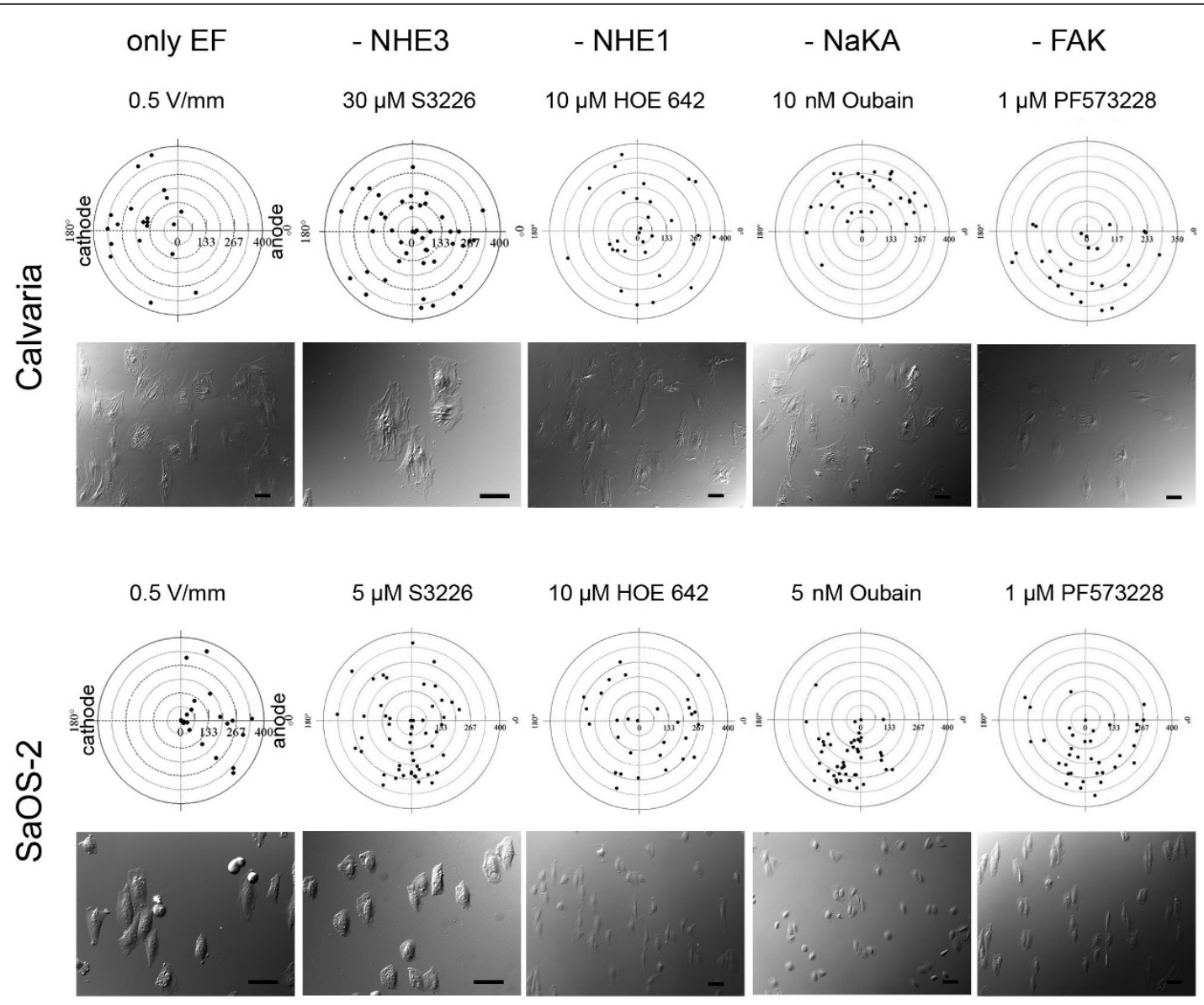

Figure 3 Polar diagrams representing the directedness of cathode (Calvaria)- and anode (SaOS-2)-directed cells in the presence of an applied EF or an EF in combination with the NHE3-, NHE1-, or NaKA-specific inhibitors, S3226, HOE 642, or oubain, respectively. FAK inhibition was used as a positive control. Each diagram represents the data from $25-45$ cells from three to five individual experiments. DIC images showing the cell shape, morphology, and orientation in the presence of EF only or with inhibitors. Bars: $50 \mu \mathrm{m}$.

electrotaxis. Taking advantage of the $\mathrm{V}_{\text {mem }}$ reporter dye, $\operatorname{DiBAC}_{4}(3)$, which is taken up by cells through the depolarized membrane, we monitored the spatiotemporal differences between the front and back ends of polarized cells during electrotaxis. In both cathode- and anodedirected cells, we observed that $\operatorname{DiBAC}_{4}(3)$ was taken up first from the rear end, whereas there was no specific direction of dye uptake in randomly migrating control cells (no EF). $V_{\text {mem }}$ kinetics collected from the selected region of interests $(\mathrm{R})$ during the time-lapse recording of the cells loaded with $\operatorname{DiBAC}_{4}(3)$ confirms that the leading edge of the cells has lower fluorescence intensity profiles (hyperpolarized) than the rear end (depolarized). However, it is also important to notice that the limitations of the technique such as fluorescence signal relevant to the morphological differences should be kept in mind when using potentiometric-, single excitation dyes.

All taken together, this observation reveals that the cell membrane had a relatively depolarized state at the rear end versus the leading edge or similarly, a relatively hyperpolarized state at the leading edge versus the rear end (Figure 6A, Additional files 5 and 6).

$\mathrm{pH}_{\mathrm{i}}$ is elevated in the membrane protrusions at the leading-edge of cathode-directed cells but not in anodedirected cells

Cathodally migrating cells, which have a well-defined polarized and elongated morphology, were observed to have bubble-shaped outward proton fluxes that are specifically located at the termini of membrane protrusions on their leading edge during electrotaxis. False colored (LUT) images shown in figure 6B represent the spatiotemporal differences in the BCECF-AM intensity profile, indicating the changes in $\mathrm{pH}_{\mathrm{i}}$. These sites were likely to be the same regions where pNHE3 had specifically accumulated on the leading edge (Figure 6B, Additional file 7). Randomly migrating control cells (no EF) also exhibited $\mathrm{pH}$ bubbles, but they were located non-specifically along the cell periphery. Conversely, there was either one 


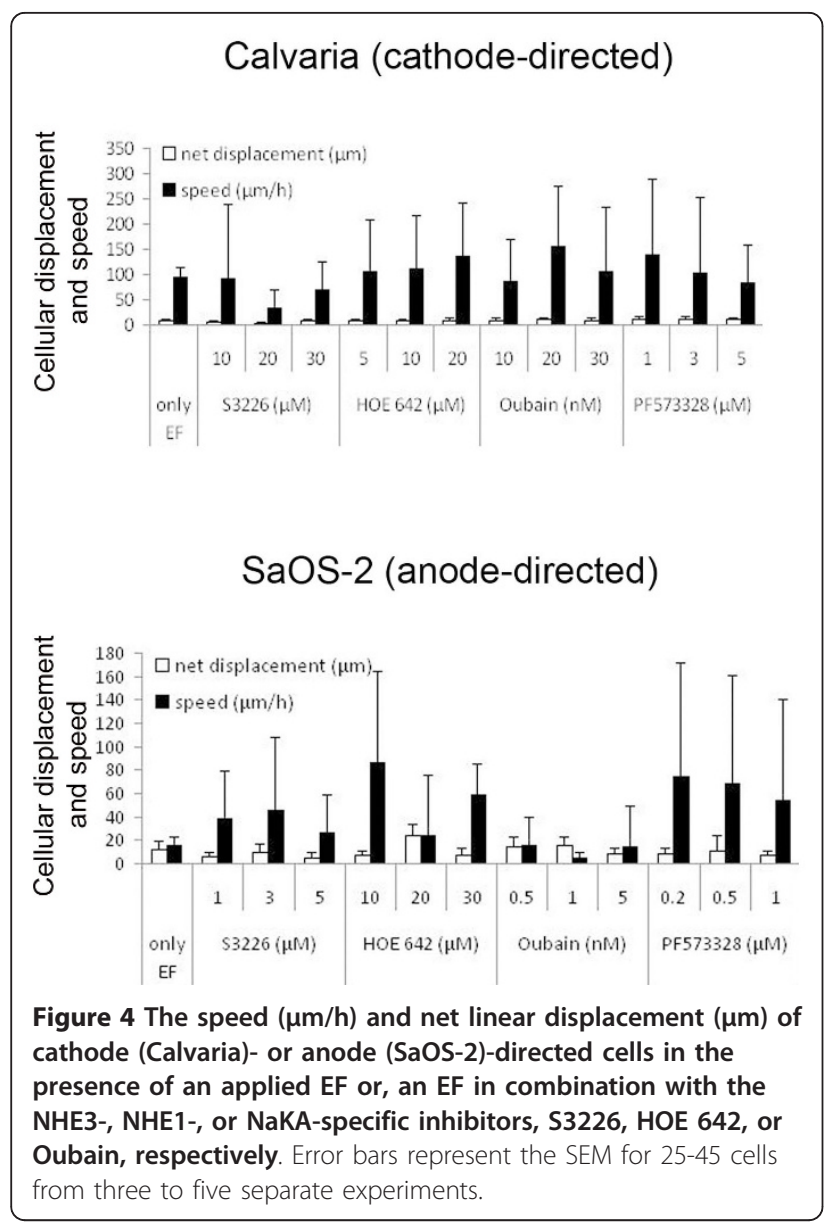

or no bubble per cell in anode-directed cells (SaOS-2) and in their controls (SaOS-2, no EF) during electrotaxis.

\section{Discussion}

This study demonstrates that NaKA, NHE3, and NHE1 are involved in cellular directedness during electrotaxis, which imply that $\mathrm{V}_{\text {mem }}$ is a spatiotemporal regulator between the leading edge and rear end. We suggest that NHE3 might be a cathode-specific sensor protein because there was an increased relocation of active NHE3 to the membrane protrusions, which was accompanied by an elevated $\mathrm{pH}_{\mathrm{i}}$ at those same sites that specifically occurred in cathode-directed cells, not in anodedirected ones, during electrotaxis. However, intracellular levels of active NHE3 and of PIP2 were increased in anode-directed cells while they were less in cathodedirected cells in comparison to randomly migrating control cells. This can result from the localized activity of NHE3 in the membrane protrusions of cathode-directed cells. Most of the data concerning NHE3 deal with its renal functions; however, few studies have demonstrated the interactions between NHE3 and cytoskeletal proteins and the related signaling pathways regarding cell motility. The actin-modifying agents cytochalasin and latrunculin have been found to inhibit epithelial NHE3 activity, whereas the housekeeping exchanger, NHE1, has been found to be virtually affected [19]. Similarly, the cytoskeletal scaffolding protein ezrin has been shown to regulate NHE3 translocation in Caco-2 cells [20]. Consistent with these studies, we demonstrate significant colocalizations of active NHE3 with the filopodia marker protein $ß$-actin on the leading-edge of both anode- and cathode-directed cells. The distinct patchy pattern of NHE3 may depend on the activity of NHE3 because only active NHE3 shows this pattern and, this activity seems to be specialized in the filopodia where $B$ actin is present. This might be due to the close interaction of these two proteins during cell migration. Additionally, the quenched directedness in the presence of the NHE3-specific inhibitor S3226 supports the idea that NHE3 is involved in directional determination during electrotaxis. Moreover, we have shown both a physiological and mechanistic role for NHE3 in cellular motility and directedness via demonstrating the colocalization of active NHE3 outward proton fluxes accompanied $B$-actin (decreased $\mathrm{pH}_{\mathrm{i}}$ ) at the leading edge of directionally migrating cells.

In contrast to NHE3, neither the expression nor the distribution of NHE1 was affected in directionally migrating cells during electrotaxis. Although the pharmacological inhibition of NHE1 via the use of HOE 642 prevented the cells from perceiving their direction which they normally would during electrotaxis, this phenomenon might be due to one of its other crucial functions as a housekeeping protein, e.g. the regulation of intracellular $\mathrm{pH}$ and volume, or to its roles in cell migration [21-25]. Therefore, we conclude that NHE1 is not specifically involved in determining the direction of migration, but instead, maintains the overall $\mathrm{pH}_{\mathrm{i}}$, volume, and osmotic balance, which is crucial to cellular physiology during directed cell motility.

The relatively hyperpolarized cell membrane on the leading edge versus the rear end of the cells, regardless of their direction, can be explained by the relocation of NaKA from the cytoplasm to the leading edge in both cathode- and anode-directed cells during electrotaxis. NaKA is a major regulator of $\mathrm{V}_{\mathrm{mem}}$ and maintains the resting membrane potential by transporting two $\mathrm{K}^{+}$ions in and three $\mathrm{Na}^{+}$ions out of the cell. If the cell membrane is hyperpolarized on the leading edge, more NaKA needs to be recruited to those sites in order to reduce the excessive positive charges on the membrane and to bring $\mathrm{V}_{\text {mem }}$ level down to its resting potential. NaKA has already been shown to be exponentially activated as a function of the $\mathrm{V}_{\mathrm{mem}}$, which in turn leads to a hyperpolarized cell membrane [26,27]. Independent of its role in ion transport, evidence exists for the involvement of NaKA in PI3K signaling and cell motility [28], in 


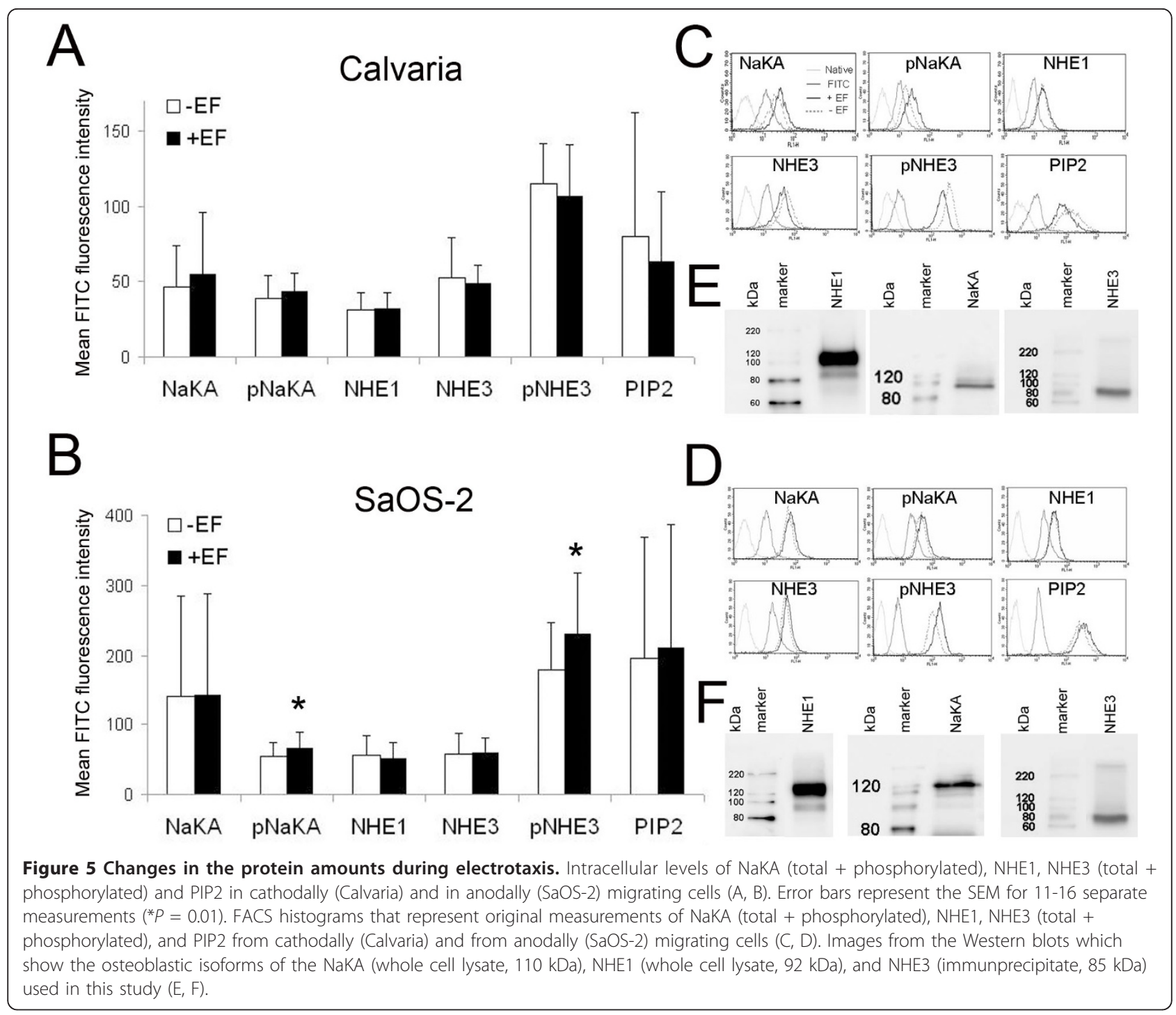

epithelial polarization and the suppression of invasion [29], and for its interaction with actin [30]. Moreover, $\mathrm{V}_{\text {mem }}$ itself has already been shown to induce cytoskeletal modifications in F-actin, microtubules, and vinculin [31] and, in adherens junctions [32] in endothelial cells. Our study demonstrates that NaKA colocalizes with the focal adhesion marker protein vinculin at the leading edge of both anode- and cathode-directed cells, and its inhibition by oubain quenches cellular directedness. This suggests that NaKA is involved in focal adhesion turnover, and, hence, the inhibition of its activity interferes with directional cell motility. Moreover, considering that the cell membrane is only hyperpolarized on the front and depolarized only on the back, regardless of the migration direction, $\mathrm{V}_{\text {mem }}$ seems to maintain the persistent directedness rather than to perceive the direction by regulating the spatiotemporal NaKA mobility between the leading edge and the rear end of cells during directional cell motility. Additionally, both the total and phosphorylated NaKA levels were increased in cathode-directed (Calvaria) cells, which indicate that both the expression and the activity of the protein were elevated in those cells during electrotaxis. In contrast, in anode-directed (SaOS2) cells, only the activity of the protein was increased, whereas the total level of the protein was not affected. These data indicate that NaKA activity is required in both anode- and cathode-directed cells.

Cellular speed was dramatically increased, especially in anode-directed cells, when NHE3 (300\%) or NHE1 $(500 \%)$ as well as FAK (500\%, control) activity was inhibited. This suggests a motility suppressor role for both NHE3 and NHE1 in these cells during directional motility. An NHE inhibition-induced reduction in cell adhesion and motility has been reported in various cell types [33-35]. These studies state that the local extracellular $\mathrm{pH}$ levels at focal adhesion sites modulate the 


\section{A}
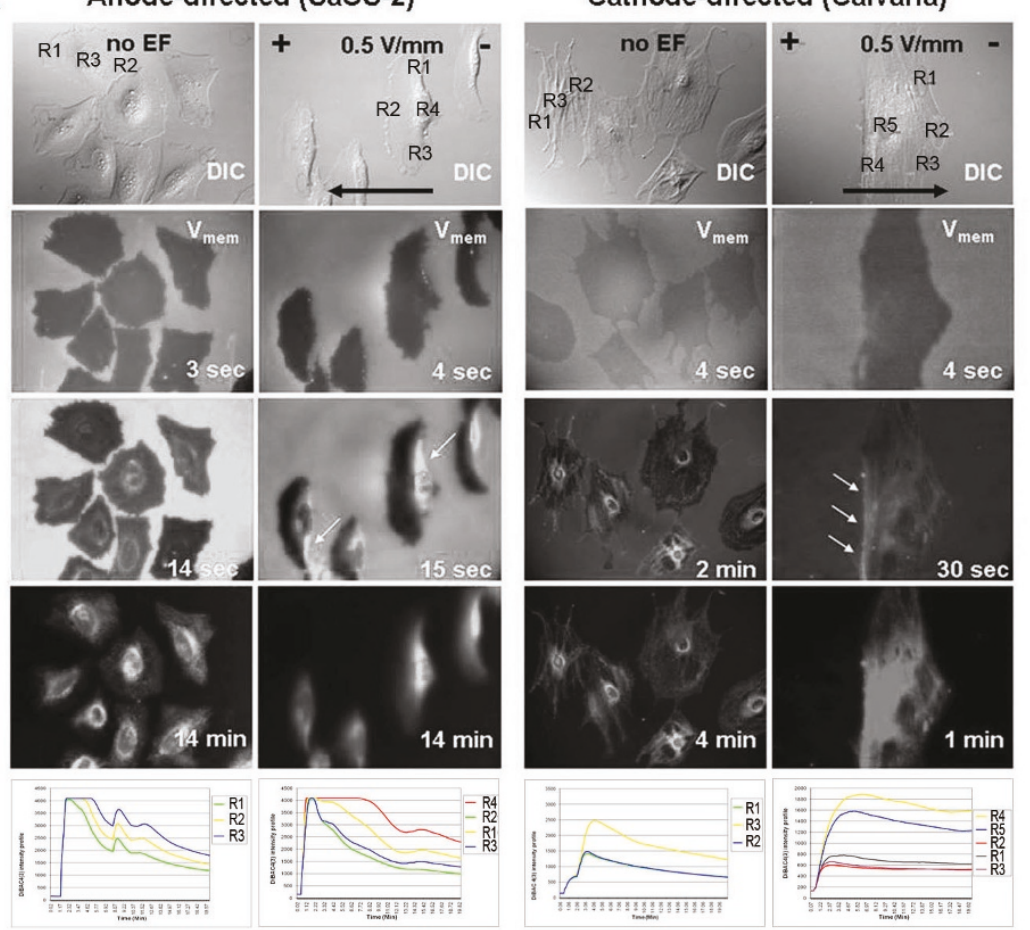

\section{Cathode-directed (Calvaria)}
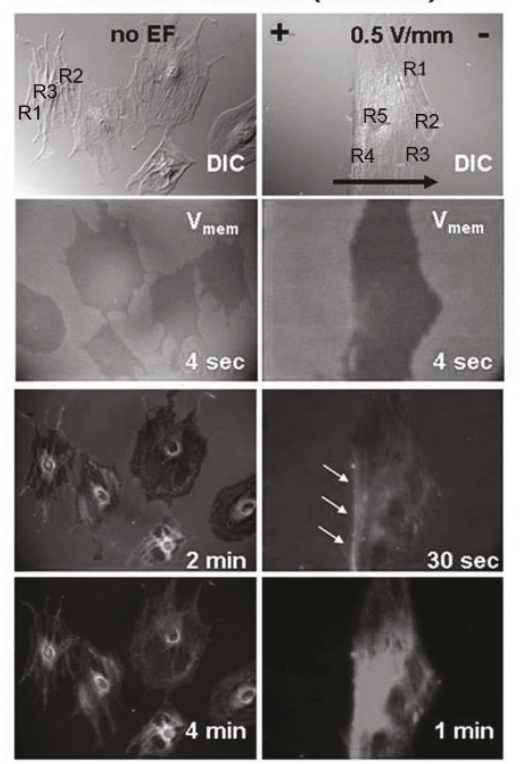

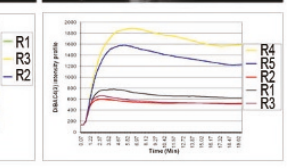





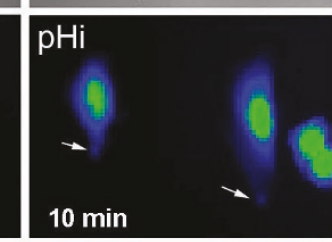

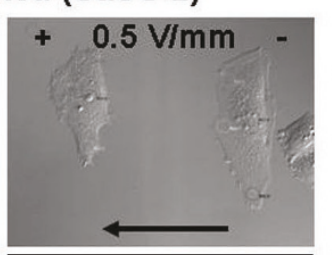

\section{low high}

\section{Cathode-directed (Calvaria)}


$4 \mathrm{~min}$

Figure 6 Spatiotemporal differences in $\mathrm{V}_{\text {mem }}$ between the leading edge and the rear end in cathode (Calvaria)- or anode (SaOS-2)directed cells. Fluorescence images showing the uptake of $V_{\text {mem }}$ reporter dye $\operatorname{DiBAC}_{4}(3)$ at different time points after addition to the cells.

White arrows in the second row point to the depolarized cell membrane, wherein the anionic dye first enters from sites that have a decreased number of negative charges (depolarized) at the rear end in both anode (left)- and cathode (right)-directed cells (A). pH bubbles (white arrows), especially recognizable in cathode-directed cells (right column), can be observed at the termini of membrane protrusions along the cell periphery in randomly migrating, nonpolarized cells and at the leading-edge of directed, polarized cells. False colored (LUT) images generated from ratiometric images showing cells loaded with BCECF-AM, a pH reporter, ratiometric dye (B). In both (A) and (B), the first row shows DIC images of the cells during random (no EF) and directed $(0.5 \mathrm{~V} / \mathrm{mm})$ migration. Black arrows in the DIC images indicate the direction of migration. 
strength of the cell adhesion and thereby migration of the cells on a collagen I matrices. An increase in NHE1 activity (more protons) was also observed to result in tighter adhesion and decreased cell migration, whereas a lack of protons, due to low NHE1 activity, prevented adhesion and migration. Further studies should elucidate the exact mechanisms of interaction between NHE3 activity $\left(\mathrm{pH}_{\mathrm{i}}\right)$ and filopodia formation so as to understand its physio-mechanical roles in persistent, directional cell motility.

In contrast to NHE3 and NHE1, the pharmacological inhibition of NaKA using oubain divergently decreased and increased the speed of anode- and of cathode-directed cells by $71 \%$ and an increase by $67 \%$, respectively. It has already been reported that independent of its role in ion transport, NaKA has various other functions in cells [36], including its role as a motility suppressor function in MDCK carcinoma cells [28]; however, the reason why NaKA activity divergently affects anode- and cathodedirected cells divergently in terms of cellular speed remains unknown and requires further investigation.

\section{Conclusions}

Our study shows that NHE3, NHE1, and NaKA, in combination with spatiotemporal changes in $\mathrm{V}_{\text {mem }}$ between the leading edge and the rear end, are required for the determination of cellular directedness during electrotaxis. Moreover, among these, NHE3 has unique responses that preferentially occur in cathode-directed cells, which suggests that it is a promising candidate for cathode-specific directional sensing (Figure 7). However, our interpretation would merit further testing, using $\mathrm{V}_{\text {mem }}$ measurements through different tools to overcome the limitations of the technique used in this study. These data can be useful for further studies in which cell guidance is an important factor.

\section{Methods}

Osteoblast cell culture

Primary osteoblastic cells were isolated from fetal rat calvaria as previously described [37]. Cultures were maintained in DMEM/Ham's F12 media (Gibco BRL, Karlsruhe/Germany) that contained $12 \%$ fetal calf

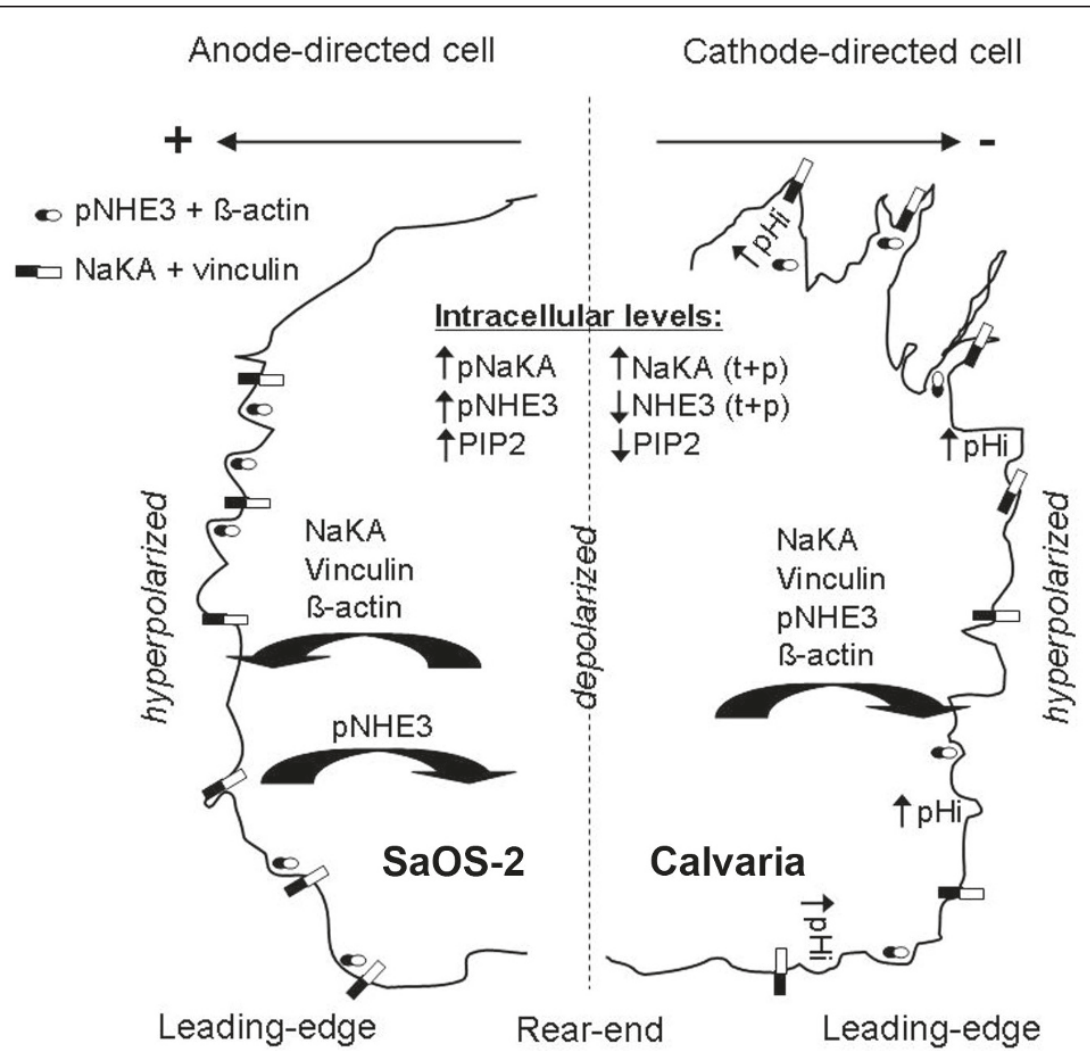

Figure 7 A schematic of NaKA and NHE3 activity on the leading edge of cathode (Calvaria)- or anode (SaOS-2)-directed cells during electrotaxis. Cells are polarized with a well-defined front and back during electrotaxis. Independent from the migration direction, NaKA is relocated from the cytoplasm to the protrusions, wherein its enhanced activity on the leading edges of cells and results in a hyperpolarized cell membrane, whereas the rear end membrane remains depolarized. Active NHE3 and consistently elevated pHi levels appear to be preferentially localized to the protrusions at the leading edges of cathode-directed cells, but not in anode-directed cells, which indicates a cathode-specific directional sensing. At the mechanical level, the colocalization of NaKA with vinculin at focal adhesion sites and the colocalization of active NHE3 with the filopodia marker B-actin suggest a direct action for NaKA and for NHE3 in adhesion turnover and in filopodia formation, respectively. 
serum, $2.3 \mathrm{mM} \mathrm{Mg}{ }^{2+}, 100 \mu \mathrm{g} / \mathrm{mL}$ penicillin, $100 \mu \mathrm{g} / \mathrm{ml}$ streptomycin sulfate, and $1.25 \% \mathrm{~L}$-glutamine at $37^{\circ} \mathrm{C}$ and in humidified $5 \% \mathrm{CO}_{2}$. Between five and seven passages were taken for the experiments. Human osteosarcoma cells (SaOS-2), which are non-transformed cells with osteoblastic properties, were obtained from the American Type Culture Collection (ATCC HTB 85) and cultured in a McCoy's 5A media (Gibco BRL, Karlsruhe/ Germany) that contained 15\% fetal calf serum and 1.25\% L-glutamine. SaOS-2 cells were used until passage number 50 because they are suspected to loose their osteoblast-phenotypic features in later passages [38]. For EF applications, $100 \mu \mathrm{L}$ of cells at a concentration of 20,000 cells $/ \mathrm{mL}$ were seeded into the channel of an ibiTreat $\mu$-Slide I (Ibidi, Munich/Germany).

\section{Electric field stimulation}

Direct current which was provided by an electrophoresis power supply (BioRad, Munich/Germany), was applied to the cells through two platinum electrodes $(0.2 \mathrm{~mm}$ in diameter, Agar scientific, Essex/UK), which were immersed in $0.9 \% \mathrm{NaCl}$ in water-filled beakers that were connected to the media-filled reservoirs of the $\mu$-Slide by two $20-\mathrm{cm}$ - long agar bridges (2\% agar in PBS). HBSS and cell culture media was used for the ion imaging and migration assays, respectively. Agar bridges were used to avoid contact between the electrode products and the cells [39]. During the experiment, field strengths were measured by a voltmeter (Voltcraft ${ }^{\mathbb{R}}$ Meßtechnik, Hirschau/Germany). The cells were exposed to a physiological range dcEF strength of $0.5 \mathrm{~V} / \mathrm{mm}$ for $5 \mathrm{~h}$ at $37^{\circ} \mathrm{C}$ in a chamber (Solent Scientific, Segensworth/UK) that covered the entire inverted microscope or in an the incubator. The same conditions were used for controls but without the application of a dcEF.

\section{Cell motility assay}

Cells were monitored via an Olympus IX81 inverted microscope that was equipped with DIC components and an integrated vital microscopy chamber (Olympus, Hamburg/Germany). An average number of 40 cells were scored for each condition. The cells were kept in their usual culture medium and exposed to EF with or without the presence of the inhibitors. Both the NHE3and NHE1-specific inhibitors, S3226 and HOE 642, were provided by Sanofi-Aventis (Frankfurt/Germany). The NaKA inhibitor, ouabain octahydrate, was purchased from Sigma Aldrich (Munich/Germany). The FAK inhibitor, PF573228, was purchased from Tocris Biosciences (Bristol/UK).

All of the collected data were analyzed with the Olympus imaging software Cell^ $R$. In order to quantify the cell speed $(\mu \mathrm{m} / \mathrm{h})$ and net linear displacement $(\mu \mathrm{m})$, time-lapse DIC videos were processed in the detecting program, TrackIT. Polar diagrams that were indicative of directedness were created using PSI-Plot (Poly Software International, New York/USA). Briefly, dots shown in the polar diagrams are corresponding to the objects (cells) whose movement has been tracked during the time course of migration. The software (TrackIT, Olympus) we used here to analyze the cell motility displays the data on cellular speed, net linear displacement and directedness separately in the graphical form. All these data series were then converted into an excel sheet using this program. Polar diagrams are widely used for presenting the data on cellular directedness in the motility assays. Polar diagrams we show in this study has been created using PSI Plot software (Poly Software International). The cellular directedness (position of the dots within $360^{\circ}$ ) in each of those diagrams is relative to the directedness of the control cells without EF; X axis having the data series analyzed from the control cells without EF and $Y$ axis having those from the cells that exposed to only EF or EF + inhibitor. Graphics that depicted the speed and distance were created in Excel. A $10 \mathrm{X}$ objective was used for visualizing the cells during the cell motility assay.

\section{Quantitative immunocytochemistry}

Cells were washed once with PBS (pH 7.4), fixed in 4\% formaldehyde $/ 0.05 \%$ glutaraldehyde for $5 \mathrm{~min}$ at room temperature (RT), permeabilized with $10 \mu \mathrm{g} / \mathrm{ml}$ digitonin (Sigma Aldrich, Munich/Germany) or with $0.5 \%$ Triton X-100 for 6 min (Sharma et al., 2008), and then blocked with $1 \%$ BSA for $20 \mathrm{~min}$. Cells were then incubated with a primary antibody overnight at $4^{\circ} \mathrm{C}$. After washing with PBS, cells were further treated with fluorescence-coupled antibodies (FITC/TexasRed) for $1 \mathrm{~h}$ at RT. Dabco-glycerin in PBS was used as a mounting solution. The sources of the antibodies included mouse monoclonal anti- $\mathrm{Na}^{+} / \mathrm{K}^{+}$-ATPase ( $\alpha 3$ subunit, 1:200, Sigma Aldrich, Munich/Germany); goat polyclonal anti-phospho $\mathrm{Na}^{+} / \mathrm{K}$ ${ }^{+}$-ATPase ( $\alpha$-Ser 943, 1:100, Santa Cruz Biotechnology Inc., Heidelberg/Germany); mouse monoclonal antiNHE-3 isoform (1:50, BD Transduction Labs, Heidelberg/Germany); mouse monoclonal anti-phospho NHE-3 (Ser 552, 1:500, Novus Biologicals, Heford/Germany); rabbit anti-human vinculin (1:200, Sigma Aldrich, Munich/Germany); mouse monoclonal anti-phosphatidylinositol 4,5-bisphosphate (PIP2, 1:200, Abcam, Cambridge/UK); rabbit polyclonal anti-beta actin (1:1000, Novus Biologicals, Heford/Germany); fluorescein-isothiocyanate (FITC)-coupled anti-mouse, anti-rabbit, or antigoat (1:500, Dianova, Hamburg/Germany); Texas Redcoupled anti-mouse and anti-rabbit (1:500, Dianova, Hamburg/Germany). A $40 \mathrm{X}$ objective was used for visualizing the cells. Image J was used to analyze the colocalization rate. For this, red:green combination of the 
channel color was set for FITC and Texas red, respectively. Then, regions of interests (ROIs) were placed where the colocalizations are observed to analyze the pixels in the defined color channels within the ROI selected. Results window displayed automatically show Pearson's correlation coefficient whose values range from 1 to -1 , where "1" indicates a perfect correlation. The plots showing the overlap rates of the proteins are generated in Excel based on their Pearson's correlation coefficient values. Relocation rate was quantified using Cell^ $R$ (Olympus) software. For quantification of relocation, three ROIs were inserted in the cytoplasm and the membrane protrusions. The mean fluorescence intensity (MFI) profiles were calculated from the quotient of the ROIs at the membrane protrusions and the ROIs in the cytoplasm. The following formula was used to calculate the relocation rate:

$$
\text { Relocation Rate }=\frac{\sum \text { MFI ROIs lamellopodia }}{\sum \text { MFI ROIs cytoplasm }}
$$

\section{FACS measurements}

Protein expression levels were analyzed by using fluorescence activated cell sorter (FACS Calibur, BD GmbH, Heidelberg/Germany). Control and EF-exposed cells were trypsinized from the Ibidi $\mu$-slides and then separately collected into the FACS tubes. Cells were washed once with DPBS ( $\mathrm{pH} 7.4$ ), fixed in $4 \%$ formaldehyde/ $0.05 \%$ glutaraldehyde at room temperature (RT) for 10 min and then centrifuged at $1200 \mathrm{rpm}$ for $4 \mathrm{~min}$. Next, the cells were permeabilized with $10 \mu \mathrm{g} / \mathrm{ml}$ digitonin for 10 minutes, centrifuged, and then blocked with $0.5 \%$ BSA for $30 \mathrm{~min}$. The cells were then incubated with a primary antibody for $1 \mathrm{~h}$ at RT. After washing with DPBS, the cells were then treated with FITC-conjugated antibodies for $1 \mathrm{~h}$ at RT. Finally, the cells were washed with DPBS and FACS was used to measure their relative fluorescence intensity. For each analysis, 5,000 cells were counted. For the detection of proteins of interest, different sources of antibodies were used, including mouse monoclonal anti- $\mathrm{Na}^{+} / \mathrm{K}^{+}$-ATPase ( $\alpha 3$ subunit, 1:100, Sigma Aldrich, Munich/Germany); goat polyclonal anti-phospho $\mathrm{Na}^{+} / \mathrm{K}^{+}$-ATPase ( $\alpha$-Ser 943, 1:100, Santa Cruz Biotechnology Inc., Heidelberg/Germany); mouse monoclonal anti- $\mathrm{Na}^{+} / \mathrm{H}^{+}$exchanger (NHE)-1 isoform (1:50, BD Transduction Labs, Heidelberg/Germany); mouse monoclonal anti-NHE-3 isoform (1:50, BD Transduction Labs, Heidelberg/Germany); mouse monoclonal anti-phospho NHE-3 (Ser 552, 1:200, Novus Biologicals, Heford/Germany); mouse monoclonal antiphosphatidylinositol 4,5-bisphosphate (PIP2, 1:100, Abcam, Cambridge/UK) and fluorescein-isothiocyanate
(FITC)-coupled anti-mouse and anti goat (1:100, Dianova, Hamburg/Germany).

\section{Immunoprecipitation}

Cells were washed with ice-cold PBS. Whole cell lysates were prepared with a RadioImmunoPrecipitation Assay (RIPA) buffer by gentle scraping. The RIPA buffer was prepared with $50 \mathrm{mM}$ Tris HCL at a $\mathrm{pH}$ of $8,150 \mathrm{mM}$ $\mathrm{NaCl}, 1 \% \mathrm{NP}-40$, and $0.5 \%$ sodium deoxycholate. Preclear lysates were incubated with either mouse monoclonal anti- $\mathrm{Na}^{+} / \mathrm{K}^{+}$-ATPase ( $\alpha 3$ subunit, 1:500, Sigma Aldrich, Munich/Germany) or mouse monoclonal anti$\mathrm{Na}^{+} / \mathrm{H}^{+}$exchanger (NHE)-1 isoform (1:250, BD Transduction Labs, Heidelberg/Germany), or mouse monoclonal anti-phospho NHE-3 (Ser 552, 1:250, Novus Biologicals, Heford/Germany) for $1 \mathrm{~h}$ at RT. These lysates were further incubated with immobilized protein $\mathrm{A} / \mathrm{G}$ beads (ImmunoPure, Pierce) for $2 \mathrm{~h}$ at $4^{\circ} \mathrm{C}$. Finally, these beads were washed three times with a RIPA buffer and centrifuged at $600 \mathrm{~g}$ for $5 \mathrm{~min}$.

\section{Western Blot}

Proteins were eluted from the beads via the addition of $50 \mu \mathrm{L}$ of a $2 \mathrm{x}$ SDS-PAGE sample buffer. After heating at $95^{\circ} \mathrm{C}$ for $5 \mathrm{~min}$, proteins were separated based on their size by an SDS that contained (10\%) polyacrylamide (Roth, Karlsruhe/Germany) gels and then transferred to PVDF membranes (Millipore, Schwalbach/Germany) by the semi-dry transfer method. Membranes were blocked at RT for 30 min with TBST (TBS with $0.1 \%$ Tween20 ), which contained $5 \%$ skim milk powder. Blots were then incubated overnight at $4^{\circ} \mathrm{C}$ with primary antibodies, including mouse monoclonal anti- $\mathrm{Na}^{+} / \mathrm{K}^{+}$-ATPase $(\alpha 3$ subunit, 1:500, Sigma Aldrich, Munich/Germany), mouse monoclonal anti- $\mathrm{Na}^{+} / \mathrm{H}^{+}$exchanger (NHE)-1 isoform (1:250, BD Transduction Labs, Heidelberg/Germany), or mouse monoclonal anti-phospho NHE-3 (Ser 552, 1:250, Novus Biologicals, Heford/Germany). Blots were washed three times ( 5 min each) with TBST. For standard Western blot detection, the blots were incubated for $1 \mathrm{~h}$ at RT with an anti-mouse HRP conjugated antibody (1:2000, Amersham Biosciences, Freiburg/Germany). Afterwards, the blots were developed with chemiluminescent HRP substrate (Immobilon Western, Millipore, Schwalbach/Germany) using image readerLAS 3000 (Fujifilm, Dusseldorf/Germany).

\section{The real-time imaging of $\mathrm{pH}$ and the cell membrane potential}

Ion-specific vital dyes were used to monitor dcEF induced changes in intracellular ions and in cell membrane potential. Ratiometric dye BCECF-AM was used as a reporter dye for intracellular $\mathrm{pH}\left(\mathrm{pH}_{\mathrm{i}}\right)$ and potentiometric dye $\mathrm{DiBAC}_{4}(3)$ was used for cell membrane 
potential $\left(\mathrm{V}_{\text {mem }}\right)$. DiBAC4(3) has already been successfully used to assess the differences in the membrane potential both in vivo $[40,41]$ and in vitro $[42,43]$ - studies. Both dyes were purchased from Invitrogen (Darmstadt/Germany). After $5 \mathrm{~h}$ of EF exposure, the cells were washed once with HBSS and then loaded with $0.5 \mu \mathrm{M}$ $\mathrm{DiBAC}_{4}(3)$ in $\mathrm{HBSS}$ at $37^{\circ} \mathrm{C}$ for $30 \mathrm{~min}$. Subsequently, $\mathrm{V}_{\text {mem }}$ dye uptake and intracellular localization was monitored using an inverted microscope with the appropriate filters for the $\operatorname{DiBAC}_{4}(3)$ dye (excitation/emission; $493 \mathrm{~nm} / 516 \mathrm{~nm}$ ). $\mathrm{V}_{\text {mem }}$ kinetics were generated from the selected region of interests during the time-lapse recording of the cells loaded with $\operatorname{DiBAC}_{4}(3)$. A background region was also included in the settings during time-lapse recordings for background subtraction. For $\mathrm{pH}_{\mathrm{i}}$ monitoring, the cells were loaded with $1 \mu \mathrm{M}$ BCECF-AM dye in HBSS and merged images generated from the dual excitation (490 nm and $440 \mathrm{~nm}$, emission; $530 \mathrm{~nm}$ ) were recorded using an inverted microscope with the specific filters for BCECF-AM. $\mathrm{pH}$ bubbles were observed at the leading edge of the cells when the cells were exposed to further stimulation with $0.5 \mathrm{~V} /$ $\mathrm{mm} \mathrm{EF}$. The cells were monitored using the imaging software Cell^R and a 40X objective.

\section{Statistics}

Statistical calculations were performed in PSI-Plot using paired or unpaired Student's t-tests, or ANOVA. A $P<0.05$ was defined as significant (* or $\mathbb{\$}$ ). Data are presented as mean \pm SEM.

\section{Additional material}

Additional file 1: Intracellular distribution of NHE3 (TRITC-labeled) The total NHE3 is evenly distributed with slight accumulations in the membrane protrusions in both polarized (+EF, right panel) and nonpolarized (-EF, left panel) cells during cathode (Calvaria)- or anode (SaOS2)-directed motility.

Additional file 2: Intracellular distribution of NHE1 (FITC-labeled) The cellular distribution of NHE1 (left panel) is not affected during cathode (Calvaria)- or anode (SaOS-2)-directed motility (right panel).

Additional file 3: Intracellular distribution of phosphorylated NaKA

(FITC-labeled). Phosphorylated NaKA is homogenously distributed at the cell membrane during directed motility in both cathode (Calvaria)- or anode (SaOS-2)-directed cells.

Additional file 4: Intracellular distribution of PIP2 (FITC-labeled). PIP2 localizes along the cell periphery both in anode (SaOS-2) and cathode-directed (Calvaria) cells.

Additional file 5: Time lapse imaging of $\operatorname{DiBAC}(4) 3$ uptake into the cathode-directed (Calvaria) cells. The dye enters into the cells through the depolarized membrane at the rear end of the polarized cells regardless of their direction. Images were collected every $35 \mathrm{sec}$ for 20 min using inverted fluorescence microscopy.

Additional file 6: Time lapse imaging of DiBAC(4)3 uptake into the anode-directed (SaOS-2) cells. The dye enters into the cells through the depolarized membrane at the rear end of the cells regardless of their direction. Images were collected every $35 \mathrm{sec}$ for $20 \mathrm{~min}$ using inverted fluorescence microscopy.
Additional file 7: Time lapse imaging of $\mathrm{H}^{+}$fluxes at the cell periphery. $\mathrm{pH}$ bubble formation at the termini of membrane protrusions on the leading-edge of cathode-directed (Calvaria) cells during electrotaxis. Cells were loaded with BCECF-AM, a pH reporter, ratiometric dye. Images were collected every 9 sec for 25 min using inverted fluorescence microscopy.

\section{Acknowledgements}

We thank L. Aschenbach and K. Sack for help with the immunocytochemistry studies, K. Pehlke for skillful technical assistance, and Dr. C. Roehlecke for helpful advices on the flow cytometry. We also thank Dr. J. Pünter (Sanofi-Aventis) for providing the NHE-specific inhibitors used in this study.

This work was supported by German Research Foundation (DFG) in context of the Graduiertenkolleg "Nano- und Biotechniken für das Packaging elektronischer Systeme" (DFG-GK "NBP", Scholarship to S. Perike) and by MeDDrive-33 Program (Medical Faculty, Technical University of Dresden, Özkucur 60.194).

\section{Authors' contributions}

SP and NÖ carried out the immnunocytochemical, FACS, cell motility and time-lapse video recording studies. PS is carried out the inhibition and western blot studies. NÖ, SP and PS analyzed the data. NÖ performed the statistical analysis. NÖ and SP drafted and wrote the manuscript. NÖ and RHWF conceived, designed and coordinated the study. All authors read and approved the final manuscript.

Received: 30 July 2010 Accepted: 22 January 2011

Published: 22 January 2011

\section{References}

1. Petrie RJ, Doyle AD, Yamada KM: Random versus directionally persistent cell migration. Nat Rev Mol Cell Biol 2009, 10(8):538-549.

2. Huang L, Cormie P, Messerli MA, Robinson KR: The involvement of Ca2+ and integrins in directional responses of zebrafish keratocytes to electric fields. J Cell Physiol 2009, 219(1):162-172.

3. Pu J, Zhao M: Golgi polarization in a strong electric field. J Cell Sci 2005, 118(6):1117-1128.

4. Yan X, Han J, Zhang Z, Wang J, Cheng Q, Gao K, Ni Y, Wang Y: Lung cancer A549 cells migrate directionally in DC electric fields with polarized and activated EGFRs. Bioelectromagnetics 2009, 30(1):29-35.

5. Wei JF, Wei L, Zhou X, Lu ZY, Francis K, Hu XY, Liu Y, Xiong WC, Zhang X, Banik NL, et al: Formation of Kv2.1-FAK complex as a mechanism of FAK activation, cell polarization and enhanced motility. J Cell Physiol 2008, 217(2):544-557.

6. Schwab A, Wulf A, Schulz C, Kessler W, Nechyporuk-Zloy V, Romer M, Reinhardt J, Weinhold D, Dieterich P, Stock C, et al: Subcellular distribution of calcium-sensitive potassium channels (IK1) in migrating cells. J Cell Physiol 2006, 206(1):86-94.

7. Goswami C, Hucho T: TRPV1 expression-dependent initiation and regulation of filopodia. J Neurochem 2007, 103(4):1319-1333.

8. Djamgoz MBA, Mycielska M, Madeja Z, Fraser SP, Korohoda W: Directional movement of rat prostate cancer cells in direct-current electric field: involvement of voltagegated $\mathrm{Na}+$ channel activity. J Cell Sci 2001, 114(14):2697-2705

9. Gao Z, Ruden DM, Lu X: PKD2 cation channel is required for directional sperm movement and male fertility. Curr Biol 2003, 13(24):2175-2178.

10. Auguste KI, Jin S, Uchida K, Yan D, Manley GT, Papadopoulos MC, Verkman AS: Greatly impaired migration of implanted aquaporin-4deficient astroglial cells in mouse brain toward a site of injury. Faseb $J$ 2007, 21(1):108-116.

11. Morokuma J, Blackiston D, Adams DS, Seebohm G, Trimmer B, Levin M: Modulation of potassium channel function confers a hyperproliferative invasive phenotype on embryonic stem cells. Proc Natl Acad Sci USA 2008, 105(43):16608-16613

12. Wei $C$, Wang $X$, Chen M, Ouyang K, Song LS, Cheng H: Calcium flickers steer cell migration. Nature 2009, 457(7231):901-905.

13. Ozkucur N, Monsees TK, Perike S, Do HQ, Funk RH: Local calcium elevation and cell elongation initiate guided motility in electrically stimulated osteoblast-like cells. PLoS One 2009, 4(7):e6131. 
14. Koliakos G, Paletas K, Kaloyianni M: NHE-1: a molecular target for signalling and cell matrix interactions. Connect Tissue Res 2008, 49(3):157-161.

15. Kaplan $\mathrm{JH}$ : A moving new role for the sodium pump in epithelial cells and carcinomas. Sci STKE 2005, 2005(289):31.

16. Hayashi H, Szaszi K, Grinstein S: Multiple modes of regulation of $\mathrm{Na}+/ \mathrm{H}+$ exchangers. Ann N Y Acad Sci 2002, 976:248-258.

17. Geering K: Functional roles of Na, K-ATPase subunits. Curr Opin Nephrol Hypertens 2008, 17(5):526-532

18. Schwab A: Ion channels and transporters on the move. News Physiol Sci 2001, 16:29-33.

19. Kurashima K, D'Souza S, Szaszi K, Ramjeesingh R, Orlowski J, Grinstein S: The apical $\mathrm{Na}(+) / \mathrm{H}(+)$ exchanger isoform NHE3 is regulated by the actin cytoskeleton. J Biol Chem 1999, 274(42):29843-29849.

20. Zhao H, Shiue H, Palkon S, Wang Y, Cullinan P, Burkhardt JK, Musch MW, Chang EB, Turner JR: Ezrin regulates NHE3 translocation and activation after Na+-glucose cotransport. Proc Natl Acad Sci USA 2004, 101(25):9485-9490.

21. Denker SP, Barber DL: Cell migration requires both ion translocation and cytoskeletal anchoring by the $\mathrm{Na}-\mathrm{H}$ exchanger NHE1. J Cell Biol 2002, 159(6):1087-1096.

22. Stock $\mathrm{C}$, Schwab A: Role of the $\mathrm{Na} / \mathrm{H}$ exchanger NHE1 in cell migration. Acta Physiol (Oxf) 2006, 187(1-2):149-157.

23. Ritter M: Cell volume regulatory ion transport in cell migration. Contrib Nephrol 1998, 123:135-157.

24. Rosengren S, Henson PM, Worthen GS: Migration-associated volume changes in neutrophils facilitate the migratory process in vitro. Am $J$ Physiol 1994, 267(6 pt 1):C1623-1632.

25. Klein M, Seeger $P$, Schuricht $B$, Alper SL, Schwab A: Polarization of $\mathrm{Na}(+) / \mathrm{H}$ (+) and $\mathrm{Cl}(-) / \mathrm{HCO}(3)(-)$ exchangers in migrating renal epithelial cells. J Gen Physiol 2000, 115(5):599-608.

26. Huang F, Rabson D, Chen W: Distribution of the NA/K pumps' turnover rates as a function of membrane potential, temperature, and ion concentration gradients and effect of fluctuations. J Phys Chem B 2009, 113(23):8096-8102.

27. Chen W, Dando R: Membrane potential hyperpolarization in Mammalian cardiac cells by synchronization modulation of $\mathrm{Na} / \mathrm{K}$ pumps. J Membr Biol 2008, 221(3):165-173.

28. Barwe SP, Anilkumar G, Moon SY, Zheng Y, Whitelegge JP, Rajasekaran SA Rajasekaran AK: Novel role for Na, K-ATPase in phosphatidylinositol 3kinase signaling and suppression of cell motility. Mol Biol Cell 2005, 16(3):1082-1094

29. Rajasekaran SA, Palmer LG, Quan K, Harper JF, Ball WJ Jr, Bander NH, Peralta Soler A, Rajasekaran AK: Na, K-ATPase beta-subunit is required for epithelial polarization, suppression of invasion, and cell motility. Mol Biol Cell 2001, 12(2):279-295.

30. Cantiello HF: Actin filaments stimulate the $\mathrm{Na}(+)-\mathrm{K}(+)-$ ATPase. Am J Physiol 1995, 269(5 pt 2):F637-643.

31. Chifflet S, Hernandez JA, Grasso S, Cirillo A: Nonspecific depolarization of the plasma membrane potential induces cytoskeletal modifications of bovine corneal endothelial cells in culture. Exp Cell Res 2003, 282(1):1-13.

32. Nin V, Hernandez JA, Chifflet S: Hyperpolarization of the plasma membrane potential provokes reorganization of the actin cytoskeleton and increases the stability of adherens junctions in bovine corneal endothelial cells in culture. Cell Motil Cytoskeleton 2009, 66(12):1087-1099.

33. Buerke U, Pruefer D, Carter JM, Russ M, Schlitt A, Prondzinsky R, Makowski J, Rohrbach S, Niemann B, Schulze C, et al: Sodium/hydrogen exchange inhibition with cariporide reduces leukocyte adhesion via P-selectin suppression during inflammation. Br J Pharmacol 2008, 153(8):1678-1685.

34. Stock C, Gassner B, Hauck CR, Arnold H, Mally S, Eble JA, Dieterich P, Schwab A: Migration of human melanoma cells depends on extracellular $\mathrm{pH}$ and $\mathrm{Na}+\mathrm{H}+$ exchange. J Physiol 2005, 567(1):225-238.

35. Wang D, King SM, Quill TA, Doolittle LK, Garbers DL: A new sperm-specific $\mathrm{Na}+/ \mathrm{H}+$ exchanger required for sperm motility and fertility. Nat Cell Biol 2003, 5(12):1117-1122

36. Krupinski T, Beitel GJ: Unexpected roles of the Na-K-ATPase and other ion transporters in cell junctions and tubulogenesis. Physiology (Bethesda) 2009, 24:192-201.

37. Monsees TK, Barth K, Tippelt S, Heidel K, Gorbunov A, Pompe W, Funk RH: Effects of different titanium alloys and nanosize surface patterning on adhesion, differentiation, and orientation of osteoblast-like cells. Cells Tissues Organs 2005, 180(2):81-95.

38. Hausser HJ, Brenner RE: Phenotypic instability of Saos-2 cells in long-term culture. Biochem Biophys Res Commun 2005, 333(1):216-222.

39. Zhao M, Agius-Fernandez A, Forrester JV, McCaig CD: Directed migration of corneal epithelial sheets in physiological electric fields. Invest Ophthalmol Vis Sci 1996, 37(13):2548-2558.

40. Ozkucur N, Epperlein HH, Funk RH: Ion imaging during axolotl tail regeneration in vivo. Dev Dyn 239(7):2048-2057

41. Adams DS, Robinson KR, Fukumoto T, Yuan S, Albertson RC, Yelick P, Kuo L, McSweeney M, Levin M: Early, H+-V-ATPase-dependent proton flux is necessary for consistent left-right patterning of non-mammalian vertebrates. Development 2006, 133(9):1657-1671.

42. Lloyd D, Harris JC, Maroulis S, Biagini GA, Wadley RB, Turner MP, Edwards MR: The microaerophilic flagellate Giardia intestinalis: oxygen and its reaction products collapse membrane potential and cause cytotoxicity. Microbiology 2000, 146(12):3109-3118.

43. Maldonado EN, Patnaik J, Mullins MR, Lemasters JJ: Free tubulin modulates mitochondrial membrane potential in cancer cells. Cancer Res 70(24):10192-10201.

doi:10.1186/1471-2121-12-4

Cite this article as: Özkucur et al:: Persistent directional cell migration requires ion transport proteins as direction sensors and membrane potential differences in order to maintain directedness. BMC Cell Biology 2011 12:4.

\section{Submit your next manuscript to BioMed Central and take full advantage of:}

- Convenient online submission

- Thorough peer review

- No space constraints or color figure charges

- Immediate publication on acceptance

- Inclusion in PubMed, CAS, Scopus and Google Scholar

- Research which is freely available for redistribution

Submit your manuscript at www.biomedcentral.com/submit
C) Biomed Central 